\title{
Management of rabies
}

\author{
Weyer $\mathrm{J}^{1,2}$ Lucille Blumberg ${ }^{1}$ \\ ${ }^{1}$ Centre for Emerging Zoonotic and Parasitic Diseases, National Institute for Communicable Diseases of the National Health Laboratory \\ Service, South Africa \\ ${ }^{2}$ Centre for Viral Zoonoses, Department of Medical Virology, University of Pretoria, South Africa \\ Corresponding author:jacquelinew@nicd.ac.za
}

Rabies is endemic in South Africa and human rabies cases continue to be reported annually. Most human cases in South Africa are dog-transmitted. Whilst efforts are underway to control and eventually eliminate dog rabies in the country, prevention of the disease through appropriate use of rabies postexposure prophylaxis is critical to save lives. This article provides a summary of rabies in South Africa and key aspects of the prevention of the disease in exposed humans.

Keywords: rabies, human rabies, rabies vaccination, post-exposure prophylaxis

\section{Introduction}

Rabies virus infection results in a fatal encephalitis in humans and animals. The disease is caused by the rabies and other rabies-related lyssaviruses transmitted from animals to humans. ${ }^{1}$ Annually up to 59000 human cases of rabies occur around the world. ${ }^{2}$ Almost all human rabies cases are linked to domestic dog exposures. ${ }^{2}$ In South Africa, rabies has been reported in domestic dogs and other animals for many years. ${ }^{3,4}$ In the 1980s and 1990s dog and dog-transmitted human rabies were reported almost exclusively from the KwaZulu-Natal and Eastern Cape provinces. ${ }^{3}$ Since 2000, dog rabies (and consequently human rabies cases) has, however, been reported from all of the provinces except the Western Cape province. ${ }^{4-7}$ Dog rabies is reported at different frequencies and distributions within the provinces and this mirrors the ability to control the disease in different parts of the country over time. Apart from dog rabies in South Africa, the rabies virus is also reported from other reservoir species including the bat-eared fox, black-backed jackal and yellow mongoose. ${ }^{4}$ Cases of rabies have also been reported in other wildlife and also several livestock species (including cattle, goat, sheep, donkeys, horses etc.), although all of these act as deadend hosts. Human cases have rarely been associated with any of these species. ${ }^{7}$

The rabies virus is transmitted through the saliva of a rabid animal to other animals or humans. Humans are also incidental dead-end hosts of the infection and transmission from humanto-human is exceptional. The latter has been restricted to rare cases of organ transplantation from individuals that have died of undiagnosed rabies. ${ }^{8}$ The virus in the infected saliva may enter the body through any break of the skin or through infection of mucosa. The saliva may be introduced into the body through bites or nicks or through licks on broken skin or mucosa. Wounds therefore do not have to be deep or large, but potentially any contact of the virus with broken skin or the mucosa should be considered a risk for exposure.
After exposure, an incubation period of 20-90 days follows. Shorter incubation periods have been reported and usually involve cases with severe bites on the face, neck or other highly innervated areas. ${ }^{9,10}$ Incubation periods of up to several years have been reported, but very rarely. ${ }^{11}$ There is a short prodrome with very non-specific findings of fever, and, importantly, localised pain at the original wound site or sites (i.e. paraesthesia) in some patients. ${ }^{9,10}$ The acute phase of the disease then develops typically lasting no more then 7-14 days before the patient's demise..$^{9110}$ Clinically the patient may present either with the furious or paralytic forms of the disease. The former includes signs and symptoms such as hyper-salivation, confusion, delirium, hallucinations, behavioural changes, aggression, intermittent and painful spasms. Sympathetic nervous system manifestations are common. The patient is typically awake and very frightened. The course of the illness is rapidly progressive and patients deteriorate neurologically with multi-organ failure followed by death. The paralytic form may resemble Guillain-Barré syndrome with patients developing ascending paralysis (from the initial site of virus inoculation), coma and death. Management of patients should include investigations and empiric treatment for other viral and bacterial infections while attempting to confirm the diagnosis of rabies or obtain history to support the likely diagnosis of rabies. ${ }^{9,12}$ Once possible treatable causes of the patient's illness have been excluded and the diagnosis of rabies is highly likely, management of patients with suspected rabies is supportive. ${ }^{9,13}$ The experimental treatment for rabies, also referred to as the Milwaukee protocol, with very few exceptions, has not been successful and is not generally recommended. ${ }^{14}$ The focus is on keeping the patient as comfortable as possible and providing support for the family members. ${ }^{12}$

The clinical diagnosis is based on the observation of progressive encephalitis, supported by the reporting of a history of animal exposure to a confirmed rabid animal or an animal where the behaviour of the animal and circumstances of the exposure are highly suggestive of rabies. ${ }^{9}$ The differential diagnosis is expansive and includes not only other causes of viral encephalitis (for 
example herpes virus infections), bacterial meningitis including TB meningitis, tetanus but also non-infectious aetiologies such as drugs and strychnine poisoning. ${ }^{12}$ Specialised laboratory testing is always required to confirm rabies virus infection. ${ }^{9}$ Antemortem investigations to detect viral RNA in saliva, cerebrospinal fluid and/or nuchal biopsies should be attempted but lack sensitivity. ${ }^{15}$ Multiple saliva specimens should be submitted to improve sensitivity of the investigation. Serology is of limited value during rabies diagnostic investigations. The gold standard laboratory test for rabies however remains the detection of rabies virus antigen in postmortem collected brain specimens, and all attempts should be made to collect and submit such samples for investigation in patients who have died.

In South Africa, human rabies (both suspected and confirmed) is a Category I notifiable medical condition (see www.nicd.ac.za for more information). Specimens from humans with suspected human rabies are tested at the national reference laboratory of the National Institute for Communicable Diseases of the National Health Laboratory Service. Between five and 30 human rabies cases are laboratory-confirmed in South Africa every year and the number of cases directly correlates with the prevalence of rabies in domestic dogs in areas around the country. ${ }^{7}$ More than $70 \%$ of these cases were reported in children and teenagers.

\section{Management of rabies virus exposures and prevention of the disease}

Rabies virus infections can be prevented in almost $100 \%$ of exposed persons through timeous postexposure prophylaxis (PEP). This includes thorough wound washing, followed by the appropriate use of rabies vaccine and rabies immunoglobulin (RIG). The World Health Organization (WHO) published revised guidelines for rabies prevention in $2018 .^{16}$ These guidelines provide evidence-based, effective but simpler regimens with the aim of increasing access to pre- and postexposure prophylaxis that are both cost-saving and dose-sparing.

The WHO categorises exposures into three groups (no risk to absolute risk) based on a risk assessment of the exposure event, with PEP responses dictated by the category of exposure. Each case of exposure to a potentially rabid animal needs to be assessed and rabies PEP provided based on the risk assessment and likelihood of rabies exposure. Several important factors should be considered: the geographical location of the exposure (i.e. is this an area where rabies is known to occur, or possible?); the species of animal involved in the exposure (certain species are often associated with rabies whilst others are not e.g. small rodents, rarely primates although bites are common due to even minor provocation); the nature of the exposure (i.e. was this a provoked attacked or not?); and the health and vaccination status of the animal. Rabies PEP must be provided if there is any perceived risk of exposure to the virus. Rabies PEP is a lifesaving intervention since treatment of clinical rabies is not possible.

Thorough wound washing (or lavage) is a vital step in PEP. Washing with copious volumes of water and soap should be started at home and repeated at the health facility ideally with the addition of an iodine-based disinfectant or $70 \%$ ethanol.
Suturing of wounds should be discouraged and if essential, rather postponed until after RIG infiltration. Tetanus booster vaccination and antibiotics are prescribed as required case-bycase.

Wound care is followed by rabies vaccination in Category II (intermediate risk) and III (absolute risk) exposures. In South Africa, two purified, killed cell culture vaccines are available for use. These vaccines are considered safe for use in all groups including pregnant and lactating women, children, the elderly and persons suffering from acquired or innate immunodeficiencies, although efficacy in the latter group may be suboptimal. In patients with severe egg protein allergy, the use of the vaccine prepared in chick embryo should be avoided. Rabies vaccines must be administered intramuscularly into the deltoid or anterolateral thigh (in children), but will not be effective in eliciting an immune response if administered into the gluteus muscle. The modified four-dose Essen schedule (day 0, 1, 7, 14 or 28) is used in South Africa. Regimens using the intradermal (ID) route of rabies vaccine administration are employed as the preferred route for rabies vaccine administration in many countries due to costs saved. In South Africa the ID route is not yet approved for PEP. However in South Africa, the ID route of administration may be considered for pre-exposure prophylaxis (PrePEP). This will save on vaccine doses and costs when a number of travellers or persons requiring vaccination for protection against workrelated risks can be vaccinated at the same time and the ID route can be assured.

Category III exposures (i.e. exposure that results in any breach of the skin, draws any amount of blood, or exposures involving mucous membranes) require the administration of RIG in addition to the rabies vaccine. The vaccine elicits an immune response only 7-10 days after administration so in Category III exposures the urgent infiltration of RIG directly into the wound is critical to allow for rapid neutralisation of the virus. Individuals with documented immunodeficiency, such as symptomatic HIV infection, should be evaluated on a case-bycase basis and receive a complete course of PEP including RIG irrespective of category of exposure or previous vaccination history. In persons who have previously received rabies vaccine, RIG is not indicated postexposure. For PEP, two doses of rabies vaccine should be administered on days 0 and 1 to boost immunity from previous immunisation. Bat exposures should be managed as Category III exposures. Bite wounds are typically very small.

Several RIG are available and the dose of the specific product should be checked. The recommended dose of humanderived rabies immunoglobulin (HRIG- Rabigam ${ }^{\circ}$ ) is 20IU/kg whilst for equine-derived rabies immunoglobulin (ERIG) it is $40 \mathrm{lU} / \mathrm{kg}$. Anaphylaxis following ERIG administrations have been reported but are uncommon. A test dose is not required but the administration should be done in a health facility with the capability of responding to such an incident. In order to be effective, as much as possible of the immunoglobulin must be infiltrated directly into the wound. All wounds must be infiltrated and the RIG may be diluted in saline if multiple wounds are 
present and to ensure coverage of all wound sites. The 2018 WHO guidelines highlight the limited value of RIG injected intramuscularly as recommended in previous guidelines, and reiterates the importance of wound infiltration as the priority, especially when RIG supplies are limited. The use of local anaesthetic agents to facilitate the administration of RIG is discouraged and suturing of wounds should be delayed where possible to reduce the potential for virus spread. Bites on the fingers pose a high risk of rabies transmission due to the high concentration of nerves in those body parts. The volume of RIG that can be safely administered locally in these cases is limited due to the risk of a compartment syndrome.

Rabies PEP should ideally be provided as soon after exposure as possible as it acts immediately to neutralise the virus and may be life-saving. When patients present some time after the exposure event, PEP should still be administered without further delay. Regard the first day of presentation as day 0 for vaccine and RIG administration. If wounds have healed, the RIG should still be infiltrated in and around the wound site. No doubling of doses or changes to the prescribed regimen are recommended. RIG should not be administered seven days after the first vaccine dose has been administered; the initial vaccine will still be effective at this stage and the use of RIG will provide no advantage in terms of prevention.

\section{Pre-exposure prophylaxis (PrePEP) in individuals at risk of exposure}

Where dog-transmitted rabies is endemic the provision of rabies PEP should be prioritised and obstacles to access removed. ${ }^{16}$ This is a more cost-effective approach than routine PrePEP for the general population. Pre-exposure vaccination is strongly recommended for individuals at high or continual risk of exposure to rabies. These individuals may be predisposed due to their occupation, such as veterinarians and veterinary technicians, wildlife handlers, animal welfare workers and laboratory staff. Persons with hobbies such as the handling of bats should also receive PrePEP. Travellers should ideally receive PrePEP if they travel to areas where dog rabies is endemic and where access to rabies PEP may be limited or unavailable. ${ }^{16,17}$ The use of PrePEP obviates the need for postexposure RIG. The updated 2018 WHO guidelines recommend two doses of vaccine into the deltoid muscle on days 0 and $7 .{ }^{16}$ Persons who have received PrePEP must be given two booster doses of vaccine if there is exposure to a suspected rabid animal, regardless of the rabies serum antibody level or perceived immunity of the patient. These are given on days 0 and 1. Importantly, RIG should be omitted postexposure since it may depress the rapid boosting of antibodies. It is not clear how often boosters should be given in people with ongoing potential exposure; some authorities recommend boosters every three to five years, but this may be guided by rabies antibody testing, as available.

\section{CASE 1}

A 10-year-old child from Bizana, Eastern Cape province was bitten by a stray dog. A day later the child is taken to the healthcare facility by her grandmother who explained that the "dog came out of nowhere" and attacked the child. No further information was available about the dog. The child has several wounds on her arms and legs.

What is the approach for postexposure management for this case?

- Risk assessment: In this case, the incident occurred in an area known to report dog (and other animal) and human rabies cases. The nature of the attack is also important since often the exposures are clearly related to provocation such as teasing of an animal. In this case, the patient was bitten without warning and provocation. The health condition of the animal is also informative as animals with rabies appear symptomatic (e.g. drooling, aggressive, unsteady) and die in the days or up to two weeks following the exposure. If the animal appears unwell or is behaving strangely, rabies must be considered. Valid and up-to-date rabies vaccination certificates could also be considered as evidence to diminish the risk for rabies but should not be used in isolation to dismiss the need for PEP. This information was not available for this case but given the geographical location and unprovoked attack by a dog, rabies PEP must be provided.

- Exposure management: This is a Category III exposure and requires wound treatment, vaccination and infiltration of RIG. It is critical to infiltrate the RIG product in all wounds as previously described. Take steps to ensure that the patient returns for the full course of four vaccinations over the period of two weeks. The state veterinarian for the area should follow up on the incident.

\section{CASE 2}

A 20-year-old woman from Botshabelo, Free State province was attacked by the neighbour's dog when she tried to enter their property. She knew the dog and he was usually friendly. The woman sustained a deep laceration on her left calf. She presented to the clinic $\mathbf{1 4}$ days after the incident since the wound was not healing well.

What is the approach for postexposure management for this case?

- Risk assessment: Again, in this case, the incident occurred in an area known to report dog (and other animal) and human rabies cases. Although the encounter may be interpreted as provoked, it would be helpful to ascertain the health and vaccination status of the animal, especially since two weeks had passed since the incident. In the absence of such information that would point away from a rabies risk, based on the nature of the dog attack and geographical location of the incident, rabies PEP must be provided even given the delay in presentation to the health facility. If the animal is alive and well at this stage, rabies would not be likely, given that animals succumb to the infection within 14 days of the onset of first symptoms.

- Exposure management: This is a Category III exposure and requires wound treatment, vaccination and infiltration of RIG without further delay. It is critical to infiltrate the RIG product in all wounds as previously described. Take steps to ensure 
that the patient returns for the full course of four vaccinations over the period of two weeks.

\section{Conflict of interest}

\section{The authors have no conflict of interest to declare.}

\section{References}

1. International Committee on the Taxonomy of Viruses. Lyssaviruses. The 10th Report of the ICTV: https://talk.ictvonline.org/ictv-reports/ictv_ online_report/negative-sense-rna-viruses/mononegavirales/w/ rhabdoviridae/795/genus-lyssavirus [Accessed 7 January 2019].

2. Hampson $\mathrm{K}$, Coudeville L, Lembo $T$, et al. Estimating the global burden of endemic canine rabies. PLoS Negl Trop Dis 2015;9(4): p.e0001709.

3. Swanepoel R. Rabies. In: Coetzer JAW, Tustin RC, editors. Infectious diseases of livestock: with special reference to southern Africa. Cape Town: Oxford University Press; 2004. pp1121-1184.

4. Brown K. A modern plague. Rabies in South Africa, past and present. In: Mad dogs and meerkats. A history of resurgent rabies in southern Africa. 2011. Ohio University Press, Athens, pp1-19.

5. Sabeta CT, Weyer J, Geertsma $P$, et al. Emergence of rabies in the Gauteng Province, South Africa: 2010-2011. Journal of the South African Veterinary Association 2011; 84(1): doi:10.4102/jsava.v84i1.921

6. Le Roux K, Stewart D, Perrett KD, et al. Rabies control in KwaZulu-Natal, South Africa. Bulletin of the World Health Organization 2018;96(5):160.
7. Weyer J, Szmyd-Potapczuk AV, Blumberg LH, et al. Epidemiology of human rabies in South Africa, 1981-2007. Virus Research 2011;155(1):281-290.

8. Bronnert J, Wilde H, Tepsumethanon V, Lumlertdacha B, Hemachudha T. Organ transplantations and rabies transmission. J of Travel Med 2007;14(3):177-180.

9. Hemachundha $T$, Ugolini $G$, Wacharapluesadee $S$, et al. Human rabies; neuropathogenesis, diagnosis, and management. Lancet Neurol 2011;12:498-511.

10. Hemachunda T, Laothamatas J, Rupprecht CE. Human rabies: a disease of complex neuropathogenetic mechanisms and diagnostic challenges. Lancet Neurol 2002;1:101-109.

11. Johnson N, Fooks A, McColl K. Reexamination of human rabies case with long incubation, Australia. EID 2008;14(12):1950-1951.

12. Blumberg L, Weyer J, Frean J, Ogunbanjo GA. Rabies: an evidence-based approach to management. SA Fam Pract 2007;49(5):16-19.

13. Warrell $M$, Warrell $D$, Tarantola $A$. The imperative of palliation in the management of rabies encephalomyelitis. Trop Med Infect Dis 2017;2(4):52.

14. Jackson AC. Current and future approaches to the therapy of human rabies. Antiviral Research 2013;99:61-67.

15. Dacheux L, Reynes JM, Buchy $P$ et al. A reliable diagnosis of human rabies based on analysis of skin biopsy specimens. Clin Infect Dis 2008:47;1410-1417.

16. World Health Organization. Position paper on rabies vaccines. April 2018: http://apps.who.int/iris/bitstream/handle/10665/272171/WER9116. pdf [Accessed 8 January 2019].

17. World Health Organization. 2012. Vaccine preventable disease and vaccines. In: International Travel and Health: https://www.who.int/ith/ITH_EN_2012_ WEB_1.2.pdf [Accessed on 27 March 2019]. 